\title{
PERFIL SENSORIAL DE PÓ DE CACAU (Theobroma cacao L.) ALCALINIZADO'
}

\author{
Eliete da Silva BISPO ; Vera Lúcia Pupo FERREIRA; \\ Ligia Regina Radomille de SANTANA; Katumi YOTSUYANAGI
}

\section{RESUMO}

A Análise Descritiva Quantitativa(ADQ) foi empregada para caracterização das amostras de pós de cacau que representaram a amplitude do delineamento composto rotacional central 2 do processo de alcalinização dos "nibs" de cacau. As variáveis independentes foram faixas de temperatura de 60 a $120^{\circ} \mathrm{C}$, de tempo de 30 a 150 min e de concentração de $\mathrm{K}_{2} \mathrm{CO}$ de 1,22 a 4,78\%. Foram avaliadas oito amostras de pó de cacau representativas das variações de cor e aceitabilidade do total das amostras obtidas experimentalmente e duas amostras de marcas comerciais. A análise do aroma foi feita diretamente nos pós de cacau alcalinizados e a dos demais atributos na forma de bebida achocolatada ( $2 \%$ do pó de cacau e $7 \%$ de açúcar em leite desnatado esterilizado). Doze provadores selecionados com base no seu poder de discriminação, reprodutibilidade e concordância com a equipe geraram em consenso, pelo método de rede (Kelly's Repertory Grid Method), três termos descritos para o aroma (alcalino, chocolate e queimado) e doze para os achocolatados (solubilidade, marrom, marrom avermelhado, chocolate, queimado, caramelo, doce, adstringente, alcalino, amargo, salgado e corpo). As avaliações das amostras foram feitas monadicamente com três repetições e em cabines individuais. Os dados obtidos foram submetidos a ANOVA, teste de Tukey e Análise de Componentes Principais. As avaliações dos aromas dos pós de cacau mostraram relação direta entre o aroma alcalino e os teores de álcali, temperatura e tempo do processo. De modo geral, os produtos com menores concentrações de $\mathrm{K}_{2} \mathrm{CO}_{3}(1,22-3,00 \%)$ apresentaram aroma e sabor de chocolate mais fortes. Encontrou-se uma relação direta entre os teores de álcali no produto e o sabor alcalino, queimado e adstringente e uma relação inversa com a luminosidade da cor. Assim, as amostras com maior concentração de $\mathrm{K}_{2} \mathrm{CO}_{3}(4,78 \%)$, foram consideradas pela equipe sensorial, as de mais forte sabor e aroma alcalino, queimado e adstringente, assim como de cor marrom e marrom avermelhado mais escura. Todos os processos levaram à obtenção de amostras com alta solubilidade e fraco sabor amargo.

Palavras-chave: Theobroma cacao L.; Análise Descritiva Quantitativa; pó de cacau alcalinizado; bebida achocolatada.

\section{SUMMARY}

SENSORY PROFILE AND ACCEPTANCE OF ALKALINIZED COCOA (Theobroma cacao L.) POWDER. The Quantitative Descriptive Analysis (ADQ) was used aiming to show the sensory characterization of cocoa powder samples representing the range of a central composite design, 2 of the process of alkalization of the cocoa "nibs" using variables independent such as temperature $(60,72,90$ and $\left.108^{\circ} \mathrm{C}\right)$, time $(30,54,90,126$ and $150 \mathrm{~min})$ and concentration of $\mathrm{K}_{2} \mathrm{CO}_{3}(0.0,1.22,3.0$ and $4.78 \%)$. Two commercial samples of alkalinized cocoa powder were appraised. The analysis of the flavour was accomplished in the alkalinized cocoa powders and the other atributes in the chocolate beverage form ( $2 \%$ of the alkalinized cocoa powders and $7 \%$ of sugar in skimmed sterilized milk). Twelve panelists were selectedbased in their to detect setterness, reliability and agreement with the panel. The data were evaluated by means of variance analysis ANOVA for the factors sample and panelist, as well as of the interaction sample $\mathrm{x}$ panelist. The Tukey test was applied for comparison of the averages of samples at the level of $5 \%$. The principal components technique was applied to the data, obtaining the configuration of the samples considering all attributes. Roughly samples with $\mathrm{K}_{2} \mathrm{CO}_{3}$ between 1.22 to $3.0 \%$ and one of the commercial samples were considered with stronger chocolate flavour, as well as, weaker alkaline flavour and taste. Samples with $\mathrm{K} \mathrm{CO}$ with $4,78 \%$ were considered the darkest color and strongest alkaline flavour and astringency. For the chocolate beverage there was no significant difference at $5 \%$ level in relation to solubility and bitter flavor.

Keywords: Theobroma cacao L.; Quantitative Descriptive Analysis; alkalinized cocoa powder; chocolate beverage.

\section{1 - INTRODUÇÃO}

Diversos tipos de pós de cacau alcalinizados são utilizados em vários produtos tais como achocolatados, "mousses", pós para preparo de pudins, bolos, coberturas, pós para sorvetes e biscoitos, dentre outros, tornan-

Recebido para publicação em 03/05/2004. Aceito para publicação em 25/04/2005(001335).

Faculdade de Farmácia, Universidade Federal da Bahia - (UFBA). End.: Rua Barão de Geremoabo $s / n$, Campus de Ondina, CEP: 40170-210, Salvador-BA. E-mail: elietesb.ssa@ftc.br

Faculdade de Engenharia de Alimentos, Universidade Estadual de Campinas (UNICAMP), Campinas-SP.E-mail:veralpf@ig.com.br

Departamento Ciências da Vida, Curso de Nutrição, UNEB. End.: Estrada das Barreiras s/n, Narandiba/Cabula. CEP: 41195-001, Salvador-BA.E-mail: ligiarrs@bol.com.br

Instituto de Tecnologia de Alimentos (ITAL), Campinas-SP. E-mail: katumi@ital.br

A quem a correspondência deve ser enviada. do-os mais atrativos e com sabor mais agradável, além de oferecer opções para reduzir o uso de corantes sintéticos $[4,13]$.

A tecnologia de alcalinização de produtos de cacau surgiu no início do século XIX na Holanda, com o objetivo de obter produtos de melhor qualidade; desde então, tem sido amplamente estudada, pois além da fermentação e da torrefação resultarem no desenvolvimento de características desejáveis no produto final, também a alcalinização pode ser utilizada para acentuar o sabor e aroma, reduzir a acidez e adstringência e obter diferentes cores que variam de marrom amarelado ou avermelhado, chegando ao marrom escuro e até ao preto, dependendo das condições de processamento e do tipo de álcali empregado $[1,4,6]$.

Basicamente, os componentes do cacau reagem com as soluções aquosas alcalinizantes sob diferentes tempos e temperaturas na presença de $\mathrm{O}_{2}$, quando ocorrem 
as reações, resultando em alterações de cor, sabor e solubilidade $[8,11,13]$. Modificações nos diversos estágios do processo de alcalinização e utilização de vários agentes alcalinizantes têm sido testados com variações dos binômios de temperatura e tempo, visando a melhoria do processo [6].

BISPO [2] estudou o processo de alcalinização de "nibs" de cacau obtendo produtos com características diferenciadas, tendo como variáveis independentes: temperatura, tempo e concentração de carbonato de potássio. A autora obteve amostras de diferentes cores, com os valores de L* (luminosidade) variando de 19,1 a 33,1; de $a^{*}$ (vermelho) de 5,3 a 17,2 e de b* (amarelo) de 2,6 a 15,3 e com aceitabilidades (na forma de bebida achocolatada) avaliadas por meio de escala hedônica de 1 (desgostei extremamente) a 9 (gostei extremamente) pontos, que variaram de 4,6 a 8,0 para o sabor e de 5,3 a 7,7 para a cor.

Com base nos dados promissores sobre a gama de cores obtidas e aceitabilidade dos pós de cacau quando preparados na forma de bebidas achocolatadas [2], este trabalho teve como objetivo avaliar o perfil sensorial de amostras de pó de cacau obtidas pelos processos de alcalinização do "nibs" de cacau, juntamente com duas marcas comerciais.

\section{2 - MATERIAL E MÉTODOS}

\section{1 - Material}

Os "nibs" (cotilédones fragmentados de cacau) utilizados neste estudo, foram obtidos de grãos de cacau tostados e fermentados da variedade "Forastero", todos com padrão de qualidade microbiológico internacional, doados por indústria de chocolate localizada no estado da Bahia, Brasil.

\section{2 - Métodos}

\subsection{1 - Processamento}

As etapas do processo de alcalinização foram realizadas utilizando-se um reator simples, construído para este estudo, sendo provido com sistema de controle de aquecimento com vapor. O agente de alcalinização empregado foi o carbonato de potássio. A temperatura interna foi controlada instalando-se um termômetro com escala de zero a $120^{\circ} \mathrm{C}$.

Posteriormente, foi reduzida a umidade dos grãos utilizando-se ar forçado numa vazão de $200 \mathrm{~L} / \mathrm{h}$, temperatura de $70^{\circ} \mathrm{C}$ por 2 horas, resultando numa umidade final de $2 \%$. O conteúdo de gordura foi reduzido através de prensa hidráulica manual EVA com capacidade para $100 t o n / \mathrm{cm}^{2}$. Extração máxima foi obtida aumentandose a pressão gradualmente para 50 ton $/ \mathrm{cm}^{2}$ num período de 45 minutos. Uma pré-moagem foi realizada num moinho de cilindros da marca SUPREMA e a moagem final num moinho de cilindro refrigerado PILON, para obter uma granulometria de $99 \%$ de malha $200(75 \mu \mathrm{m})$ após quatro passagens pelo moinho operando com pressão máxima nos rolos.

\subsection{2 - Planejamento experimental}

Os parâmetros e as diferentes condições de processo de alcalinização dos "nibs" de cacau foram baseados na literatura e em testes preliminares para cada fator. Os níveis das variáveis independentes foram definidos da seguinte forma: temperatura de 60 a $120^{\circ} \mathrm{C}$, tempo de 30 a 150 min e concentração de carbonato de potássio de 1,22 a $4,78 \%$, este sendo o agente que deu a melhor cor e aroma nos testes preliminares.

Os ensaios experimentais foram realizados fazendose uso de um delineamento composto rotacional $2 \mathrm{com}$ 18 tratamentos, sendo 8 pontos fatoriais, 6 pontos axiais e 4 repetições no ponto central.

O planejamento citado teve por objetivo avaliar os efeitos das variáveis: temperatura, tempo e concentração de carbonato de potássio sobre as variáveis dependentes (respostas) cor e aceitabilidade sensorial.

\subsection{3 - Amostras}

Foram avaliadas sensorialmente 10 amostras de pó de cacau alcalinizado, sendo oito amostras provenientes dos experimentos de alcalinização descritos nos itens 2.2.1 e 2.2.2 (codificadas como 2; 4; 6; 7; 9; $11 ; 12$ e 17 na Tabela 1) e duas amostras de marcas comerciais (codificadas pelos números $18 \mathrm{e} 19$ na Tabela 1). As 8 amostras experimentais foram assim escolhidas por representarem a amplitude do experimento em termos de variação de cor e aceitabilidade.

TABELA 1 - Condições dos processos de alcalinização dos "nibs" para obtenção das amostras dos pós de cacau utilizadas neste estudo

\begin{tabular}{cccc}
\hline Amostras & $\mathrm{T}\left({ }^{\circ} \mathrm{C}\right)$ & $\mathrm{t}(\mathrm{min})$ & $\mathrm{K}_{2} \mathrm{CO}_{3}(\%)$ \\
\hline 2 & 108 & 54 & 1,22 \\
4 & 108 & 126 & 1,22 \\
6 & 108 & 54 & 4,78 \\
7 & 72 & 126 & 4,78 \\
9 & 60 & 90 & 3,00 \\
11 & 90 & 30 & 3,00 \\
12 & 90 & 150 & 3,00 \\
17 & 90 & 90 & 3,00 \\
18 & comercial & & \\
19 & comercial & \\
\hline $\mathrm{T}\left({ }^{\circ} \mathrm{C}\right)=$ temperatura em graus centígrados; $\mathrm{t}(\mathrm{min})=$ tempo em minutos; & $\mathrm{K}_{2} \mathrm{CO}_{3}(\%)=$ \\
concentração de carbonato de potássio.
\end{tabular}

As avaliações do aroma foram realizadas no pó de cacau e os demais atributos foram avaliados na forma de bebida achocolatada ( $2 \%$ do pó de cacau alcalinizado e $7 \%$ de açúcar em leite desnatado esterilizado).

\subsection{4 - Análise Descritiva Quantitativa (ADQ)}

A análise sensorial Descritiva Quantitativa (ADQ) foi realizada segundo o método descrito por STONE \& SIDEL [12].

- Equipe de julgadores

Foi empregada equipe constituída por 12 julgadores com idades variando de 20 a 49 anos e já participantes de equipe de Análise Descritiva Quantitativa de chocolates do Instituto de Tecnologia de Alimentos - ITAL. 


\section{- Desenvolvimento de terminologia descritiva}

Foram realizadas duas sessões para gerar uma lista de termos descritivos segundo método "Kelly's Repertory Grid Method" [9], para caracterizar as amostras em questão. Os provadores receberam as amostras aos pares, envolvendo todas as combinações possiveis, listando as similaridades e as diferenças percebidas entre as amostras apresentadas.

Com os atributos escolhidos consensualmente entre os membros da equipe após as duas sessões de discussões abertas, montou-se a ficha de avaliação das amostras, onde a intensidade dos descritores foi avaliada por meio de escalas não estruturadas de $10 \mathrm{~cm}$, ancoradas a $0,5 \mathrm{~cm}$ dos pontos extremos, da esquerda e direita, respectivamente, pelos termos "claro" e "escuro", "pouco" e "muito", "fraco" e "forte", dependendo do atributo considerado (Figura 1).

Foram levantados, pelos julgadores, 3 termos para descrever o aroma dos pós de cacau alcalinizado: alcalino, queimado, e chocolate e 12 termos divididos em quatro categorias de atributos para as bebidas achocolatadas: aparência (solubilidade, cor marrom e marrom avermelhada), sabor (chocolate, queimado, caramelo, doce, adstringência e alcalino), gosto (amargo e salgado) e textura (corpo ou sensação na boca) (Figura 1). A definição de todos os descritores é apresentada na Tabela 2.

- Treinamento e seleção da equipe de provadores

Os 12 julgadores foram submetidos a testes de seleção e treinamento em três sessões, utilizando-se cinco amostras de pó de cacau provenientes dos experimentos de alcalinização descritos nos ítens 2.2.1 e 2.2.2; a avaliação do aroma foi realizada no pó e demais atributos foram avaliados na forma de bebida achocolatada, por meio da ficha desenvolvida (Figura 1). Todos os julgadores avaliaram as cinco amostras em três repetições.

Foram selecionados os doze provadores recrutados, em função de suas capacidades para diferenciar as amostras, da repetibilidade ou consistência das respostas e do consenso de cada provador com a equipe sensorial. Foram selecionados os julgadores em função da ANOVA, com base nos valores de $\mathrm{F}_{\mathrm{m} \text {. . . } t . .}$ significativo para $\mathrm{p} \leq 0,05$

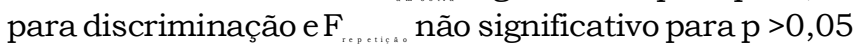
para repetibilidade e concordância dos valores das médias com as da equipe (valores de médias de cada provador na mesma ordem e próxima à média da equipe para cada atributo avaliado), segundo metodologia proposta por DAMÁSIO \& COSTEL [3] e MEILGAARD, CIVILLE \& CARR [7].

\section{- Avaliação das amostras}

As avaliações de cor foram conduzidas em cabine de iluminação Super Skyligth com iluminante C (luz do dia) e as dos demais atributos em cabines individuais iluminadas com luz vermelha para mascarar a cor das amostras e equipadas com o sistema computadorizado COMPUSENSE (1986-1992) versão 4.2 para coleta dos dados.

As amostras dos pós de cacau e das bebidas achocolatadas foram apresentadas aos 12 julgadores selecionados em copos descartáveis de polietileno branco de $50 \mathrm{~mL}$ de capacidade, codificados com números de três algarismos. A forma de apresentação foi a monádica, com cinco amostras por sessão e três repetições aleatorizadas num total de seis sessões. As bebidas achocolatadas foram servidas a temperatura de $40^{\circ} \mathrm{C}$, a fim de facilitar a avaliação dos atributos.

\section{- Análises estatísticas}

Os dados registrados no sistema computadorizado de análise sensorial foram exportados para o programa estatístico SAS [10] e submetidos à análise de variância ANOVA. Aplicou-se o teste de Tukey para comparação das médias das amostras ao nível de 5\%. Foi também realizada uma análise multivariada por meio da técnica de Componentes Principais.

TABELA 2 - Definição de descritores levantados pela equipe de julgadores e suas referências

\begin{tabular}{|c|c|c|}
\hline ATRIBUTO & DEFINICCÄO & REFERENCIAS \\
\hline \multicolumn{3}{|l|}{ AROMA } \\
\hline \multirow[t]{2}{*}{ Alcalino } & Odor produzido por solução aquosa de substâncias alcalinas (sabão) & Fraco: \\
\hline & & Forte: \\
\hline \multirow[t]{2}{*}{ Chocolate } & & Fraco: \\
\hline & & Forte: \\
\hline \multirow[t]{2}{*}{ Queimado } & & Fraco: \\
\hline & & Forte: \\
\hline \multicolumn{3}{|l|}{ APARÊNCIA } \\
\hline \multirow[t]{2}{*}{ Solubilidade } & Qualidade de solúvel, que forma solução com outra substância & Pouco: \\
\hline & & Muito: \\
\hline \multirow[t]{2}{*}{ Marrom } & & Claro: \\
\hline & & Escuro: \\
\hline \multirow{2}{*}{$\begin{array}{l}\text { Marrom } \\
\text { avermelhada }\end{array}$} & & Claro: \\
\hline & & Escuro: \\
\hline \multicolumn{3}{|l|}{ SABOR } \\
\hline \multirow[t]{2}{*}{ Chocolate } & & Fraco: \\
\hline & & Forte: \\
\hline \multirow[t]{2}{*}{ Queimado } & Sabor associado a pão, biscoito ou nozes queimados & Fraco: \\
\hline & & Forte: \\
\hline \multirow[t]{2}{*}{ Caramelo } & Gosto de açúcar derretido, lembra "toffee" & Fraco: \\
\hline & & Forte: \\
\hline \multirow[t]{2}{*}{ Doce } & & Fraco: \\
\hline & & Forte: \\
\hline \multirow[t]{2}{*}{ Adstringência } & Sensação resultante da contração da mucosa da boca & Fraco: \\
\hline & & Forte: \\
\hline \multirow[t]{2}{*}{ Alcalino } & Gosto produzido por solução aquosa de substâncias alcalinas (sabão) & Fraco: \\
\hline & & Forte: \\
\hline \multicolumn{3}{|l|}{ GOSTO } \\
\hline \multirow[t]{2}{*}{ Amargo } & & Fraco: \\
\hline & & Forte: \\
\hline \multirow[t]{2}{*}{ Salgado } & & Fraco: \\
\hline & & Forte: \\
\hline \multicolumn{3}{|l|}{ TEXTURA } \\
\hline \multirow[t]{2}{*}{ Corpo } & & Fraco: \\
\hline & & Forte: \\
\hline
\end{tabular}




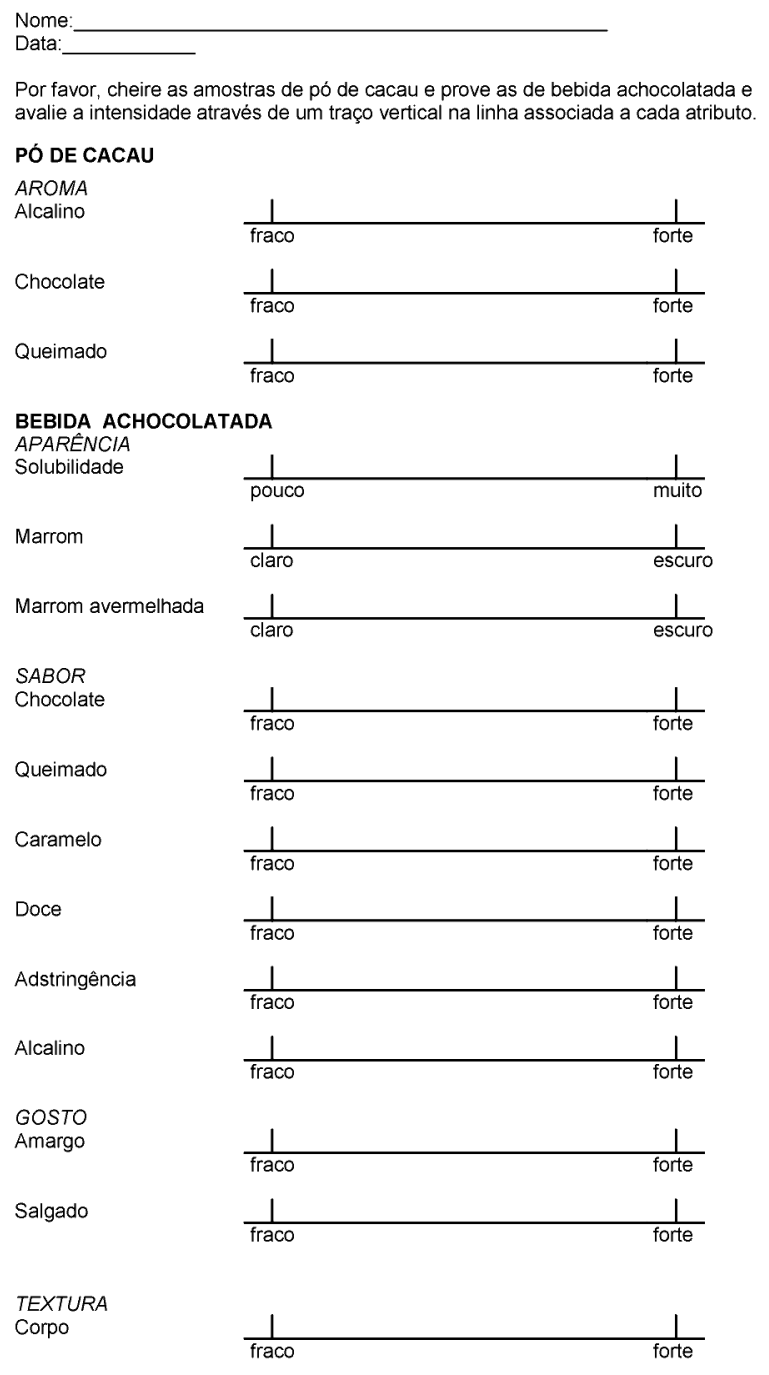

FIGURA 1 - Modelo da ficha de avaliação desenvolvida para análise sensorial

\section{3 - RESULTADOS E DISCUSSÃO}

\section{1 - Seleção dos provadores e termos descritivos}

Visto que os 12 julgadores apresentaram como resultados dos testes de seleção e treinamento valores de

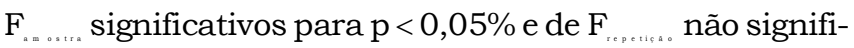
cativos para $\mathrm{p}<0,05 \%$ foi mantida a equipe para a avaliação do perfil sensorial dos pós de cacau alcalinizado. Nas comparações das médias das avaliações de cada jul- gador para cada atributo com as médias da equipe, os resultados foram considerados aceitáveis de acordo com STONE \& SIDEL [12].

\section{2 - Perfil sensorial do pó de cacau}

As amostras de pós de cacau alcalinizados diferiram significativamente entre si $(\alpha=5 \%)$ quanto ao atributo aroma (Tabela 3).

As amostras 6, 7 e 18 (comercial) foram consideradas as de aroma alcalino mais forte, estando relacionadas as duas primeiras com os tratamentos de maior teor de $\mathrm{K} \mathrm{CO}_{3}(4,78 \%$ conforme mostra a Tabela 1$)$. As amostras 2 e $4\left(1,22 \%\right.$ de $\left.\mathrm{K}_{2} \mathrm{CO}_{3}\right)$ apresentaram aroma alcalino "fraco" não mostrando diferenças significativas ao nível de $5 \%$ de probabilidade entre si e em relação às amostras 12,17 (3,00\% de $\mathrm{K} \mathrm{CO}_{z}$ ) e 19 (comercial).

Quanto ao aroma de chocolate as amostras 2, 4 $\left(1,22 \%\right.$ de $\mathrm{K} \mathrm{CO}_{2}$ ) e 19 (comercial) apresentaram aroma de chocolate mais "forte" em relação às demais, não havendo diferença significativa entre elas $(\mathrm{p} \leq 5 \%)$. As amostras 9, 11,12 e $17\left(3,0 \% \mathrm{~K} \mathrm{CO}_{3}\right)$ e a 19 (comercial) apresentaram intensidades de aroma de chocolate moderado e não houve diferença significativa entre elas ( $\mathrm{p} \leq 5 \%)$. As amostras 6 e $7\left(4,78 \% \mathrm{~K} \mathrm{CO}_{s}\right)$ receberam avaliações ao redor de 4 . A amostra 18 de marca comercial apresentou aroma de chocolate avaliado como mais "fraco" em relação às demais amostras e diferiu significativamente de todas as amostras a $\mathrm{p} \leq 0,05$.

$\mathrm{Na}$ avaliação do aroma de queimado todas as amostras apresentaram intensidade de aroma "fraco". Apenas as amostras 6 e 7 apresentaram aroma ligeiramente mais intenso.

Os dados sugerem estreita relação entre os teores de $\mathrm{K}_{2} \mathrm{CO}_{s}$ utilizados nos processos de alcalinização, aliados aos parâmetros tempo x temperatura e o aroma alcalino e de chocolate.

As amostras 2 e 4 distinguiram-se das demais por apresentar aroma de chocolate mais forte e aroma alcalino fraco e foram experimentalmente obtidas por processos de alcalinização com temperatura de $108^{\circ} \mathrm{C}$, concentração de $1,22 \%$ de $\mathrm{K} \mathrm{CO}_{s}$ e tempos de 54 e $126 \mathrm{~min}$. respectivamente (Tabela 1). As amostras 6 e 7 (4,78\% $\mathrm{K} \mathrm{CO}_{2}$ ) apresentaram aroma de chocolate fraco e aroma alcalino forte.

Pelos resultados da análise de componentes principais do aroma dos pós de cacau alcalinizados (Figura 2),

TABELA 3 - Valores médios do atributo aroma dos pós de cacau alcalinizados ( $n=12$ julgadores, $r=3$ repetições)

\begin{tabular}{|c|c|c|c|c|c|c|c|c|c|c|}
\hline Descritores & 2 & 4 & 6 & 7 & 9 & 11 & 12 & 17 & 18 & 19 \\
\hline Alcalino & $2,84 \mathrm{de}$ & $2,77 e$ & $5,09 b$ & $6,38 a$ & $4,62 \mathrm{bc}$ & $3,86 \mathrm{~cd}$ & 3,18 de & $3,55 \mathrm{de}$ & $4,85 \mathrm{bc}$ & $3,32 \mathrm{de}$ \\
\hline Chocolate & $5,48 a b$ & $6,04 a$ & $4,32 \mathrm{~cd}$ & $3,96 \mathrm{~d}$ & $4,86 \mathrm{bcd}$ & $5,21 a b c$ & $4,97 \mathrm{bc}$ & $4,73 \mathrm{bcd}$ & $2,93 \mathrm{e}$ & $5,33 a b$ \\
\hline Queimado & $1,74 \mathrm{e}$ & $2,09 \mathrm{de}$ & $3,53 a b$ & $3,72 a$ & $2,64 \mathrm{~cd}$ & $2,30 \mathrm{cde}$ & $2,27 \mathrm{cde}$ & 1,98de & $2,90 \mathrm{bc}$ & 2,03de \\
\hline
\end{tabular}

Em cada linha, médias com letras em comum não diferem significativamente $(p \leq 0,05)$ entre si, pelo teste de Tukey.

18 e 19: amostras comerciais 
observa-se que os componentes principais 1 e 2 juntos explicam $82,77 \%$ da variação total ocorrida entre as amostras. As amostras 6 e $7\left(4,78 \% \mathrm{~K} \mathrm{CO}_{3}\right)$ foram bem semelhantes entre si, caracterizando-se por apresentar intensidade de aroma alcalino e queimado mais forte e pouco aroma de chocolate.

A amostra 9 ocupa a região central do gráfico, o que leva a associação de que ela apresentou valores intermediários dos atributos de aroma de queimado, alcalino e chocolate com relação às demais amostras.

As amostras 2, $4\left(1,22 \% \mathrm{~K}_{2} \mathrm{CO}_{3}\right), 11,12,17$ (3,00\% $\mathrm{K} \mathrm{CO}_{3}$ ) e 19 (comercial) são mais caracterizadas pelo atributo aroma de chocolate. A amostra 18 (comercial) se destacou das demais por apresentar baixíssima intensidade de aroma de chocolate.

\section{3 - Perfil sensorial das bebidas achocolatadas}

Os achocolatados elaborados com as 10 amostras de pós de cacau diferiram significativamente entre si $(\alpha=5 \%)$ em relação a todos os atributos estudados, exceto o gosto amargo (Tabela 4).

As avaliações da solubilidade indicaram que todas as amostras foram "muito" solúveis (Tabela 4). Estes dados confirmam o relatado por KATTEMBERG [4], de que os pós alcalinizados são mais rapidamente dispersiveis do que os não alcalinizados, quando aplicados em alimentos.

O atributo cor foi consideravelmente influenciado pela concentração de carbonato de potássio e pela temperatura do processo. A amostra $6\left(4,78 \% \mathrm{~K} \mathrm{CO}_{2}, \mathrm{~T}=108^{\circ} \mathrm{C}\right.$, $\mathrm{t}=54 \mathrm{~min}$ ) foi avaliada como sendo de cor marrom mais escura, diferindo significativamente ( $\mathrm{p} \leq 5 \%$ ) das demais amostras. A amostra 7 (4,78\% $\mathrm{K}_{2} \mathrm{CO}_{3}, \mathrm{~T}=72^{\circ} \mathrm{C}, \mathrm{t}=126$

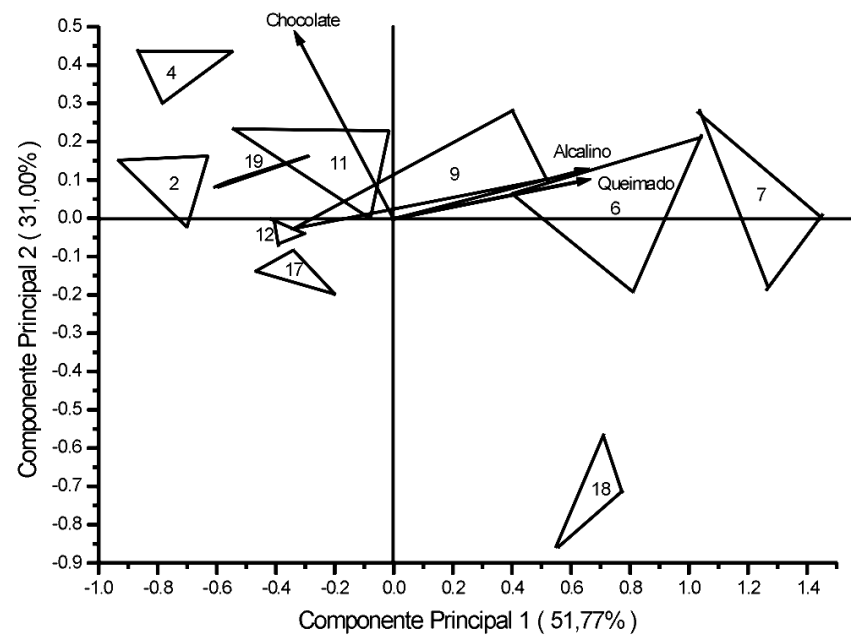

FIGURA 2 - Projeção bidimensional da análise de componentes principais da avaliação do aroma das amostras do pó de cacau alcalinizados

min) apresentou cor marrom avermelhada mais escura, diferindo significativamente $(\mathrm{p} \leq 5 \%$ ) das demais amostras. Ficou demonstrado que os experimentos com elevadas concentrações de álcali, temperaturas mais baixas $\left(<72^{\circ} \mathrm{C}\right)$ e tempos mais altos ou com elevadas concentrações de álcali e temperaturas mais altas $\left(>108^{\circ} \mathrm{C}\right)$ e tempos não tão altos apresentaram amostras de pós de cacau mais escuros. Há uma relação inversa entre os teores de álcali e a luminosidade da cor, ou seja, baixas concentrações de álcali, mesmo com elevadas temperaturas de processo $\left(>108^{\circ} \mathrm{C}\right)$ resultaram em pós de cacau com alta luminosidade (mais claros), exemplificado pela amostra 2 .

TABELA 4 - Valores médios dos atributos sensoriais das bebidas achocolatadas elaboradas com as amostras de pós de cacau ( $\mathrm{n}=12$ julgadores, $r=3$ repetições)

\begin{tabular}{|c|c|c|c|c|c|c|c|c|c|c|}
\hline \multicolumn{11}{|c|}{ ACHOCOLATADOS } \\
\hline Descritores & 2 & 4 & 6 & 7 & 9 & 11 & 12 & 17 & 18 & 19 \\
\hline Solubilidade & $6,57 a b$ & $5,90 \mathrm{~b}$ & $6,13 b$ & $5,98 \mathrm{~b}$ & $6,18 b$ & $6,35 a b$ & $6,29 a b$ & $6,46 a b$ & $5,99 \mathrm{~b}$ & $6,98 a$ \\
\hline Marrom & $3,65 f$ & $5,61 \mathrm{~cd}$ & $7,57 \mathrm{a}$ & $6,57 \mathrm{~b}$ & $4,84 \mathrm{e}$ & $4,91 \mathrm{de}$ & $4,69 \mathrm{e}$ & $5,96 \mathrm{bc}$ & $5,89 \mathrm{~b} \mathrm{c}$ & $4,46 \mathrm{e}$ \\
\hline $\begin{array}{c}\text { Marrom } \\
\text { avermelhado }\end{array}$ & $2,33 d$ & $3,33 \mathrm{c}$ & $4,95 \mathrm{~b}$ & $6,37 a$ & $4,70 \mathrm{~b}$ & $3,38 \mathrm{c}$ & $3,27 \mathrm{c}$ & $5,39 \mathrm{~b}$ & $5,40 \mathrm{~b}$ & $2,39 d$ \\
\hline Chocolate & $5,57 a b$ & $5,67 a b$ & $4,79 \mathrm{~b}$ & $4,83 \mathrm{~b}$ & $5,50 a b$ & $5,80 a$ & 5,76 a & $5,57 a b$ & $3,51 \mathrm{c}$ & $5,82 a$ \\
\hline Queimado & $1,77 \mathrm{~d}$ & $2,79 a b c$ & $3,36 a b$ & $3,38 a$ & $2,56 a b c$ & $2,10 \mathrm{~cd}$ & $2,16 \mathrm{~cd}$ & $2,34 \mathrm{c}$ & $2,54 \mathrm{bc}$ & $2,17 \mathrm{~cd}$ \\
\hline Caramelo & 3,69 a & $3,60 \mathrm{a}$ & $3,29 a b$ & $3,22 a b$ & $3,24 a b$ & $3,41 \mathrm{a}$ & $3,37 \mathrm{ab}$ & $3,06 a b$ & $2,64 b$ & $3,32 a b$ \\
\hline Doce & $4,47 \mathrm{a}$ & $2,27 \mathrm{c}$ & $3,69 \mathrm{~b}$ & $4,05 a b$ & $4,02 a b$ & $4,31 a b$ & $4,18 a b$ & $4,13 a b$ & $3,69 b$ & $4,26 a b$ \\
\hline Adstringente & $2,94 \mathrm{~b}$ & $2,91 \mathrm{~b}$ & $3,71 a b$ & $4,14 a$ & $3,43 a b$ & $3,14 b$ & $2,88 \mathrm{~b}$ & $2,99 \mathrm{~b}$ & $3,58 a b$ & $3,25 b$ \\
\hline Alcalino & $2,37 d$ & $2,79 \mathrm{bcd}$ & $3,97 a b$ & $4,40 a$ & $3,00 \mathrm{bcd}$ & $2,81 \mathrm{bcd}$ & $2,55 \mathrm{~cd}$ & $2,81 \mathrm{bcd}$ & $3,52 \mathrm{bc}$ & $3,03 \mathrm{bcd}$ \\
\hline Amargo & $2,28 a$ & $2,54 a$ & $3,00 \mathrm{a}$ & $3,00 a$ & $2,56 a$ & $2,48 a$ & $2,48 a$ & $2,45 a$ & $2,80 a$ & $2,65 a$ \\
\hline Salgado & $1,63 b$ & $1,77 \mathrm{~b}$ & $2,05 a b$ & $2,39 a$ & $1,85 b$ & $1,87 b$ & $1,79 \mathrm{~b}$ & $1,82 \mathrm{~b}$ & $2,09 a b$ & $1,73 b$ \\
\hline Corpo & $4,85 \mathrm{bc}$ & $5,55 a b$ & $5,73 a$ & $5,79 a$ & $5,20 a b c$ & $5,48 a b$ & $5,21 a b c$ & $5,59 \mathrm{a}$ & $4,76 \mathrm{c}$ & $5,22 a b c$ \\
\hline
\end{tabular}

Em cada linha, médias com letras em comum não diferem significativamente ( $\leq \leq 0,05)$ entre si, pelo teste de Tukey.

18 e 19: amostras comerciais 
As amostras 2, 4, 9, 11, 12 e 17, que foram tratadas com baixas concentrações de $\mathrm{K} \mathrm{CO}_{s}$, e a 19 (marca comercial) foram consideradas as de sabor mais forte de chocolate, recebendo médias entre 5,5 e 5,8 (Tabela 4). As amostras 6 e 7 (tratadas com altas concentrações de $\mathrm{K}_{2} \mathrm{CO}_{3}$ ) foram consideradas de sabor moderado de chocolate, recebendo médias ao redor de 4,8 (Tabela 4). Demonstrando que no processo de alcalinização se obtém sabor de chocolate mais forte com concentrações mais baixas $(<3,0 \%)$ de carbonato de potássio e temperatura na faixa de $90^{\circ} \mathrm{C}$ a $108^{\circ} \mathrm{C}$. A amostra 18 (marca comercial) apresentou sabor de chocolate mais fraco, diferindo significativamente das demais.

Com respeito às notas de sabor alcalino, as amostras 2 e 4 (amostras tratadas com $1,22 \%$ de $\mathrm{K} \mathrm{CO}$ ) apresentaram as menores notas e as amostras 6 e 7 as maiores (amostras tratadas com $4,78 \%$ de $\mathrm{K} \mathrm{CO}_{3}$ ), porém próximas ao "fraco" da escala utilizada (Figura 1). Com relação ao sabor queimado as amostras apresentaram médias entre 2,0 e 3,0 (Tabela 4), próximas ao "fraco" da escala utilizada (Figura 1).

Todas as amostras apresentaram sabor de caramelo próximo ao "fraco". As amostras 2 e 4 avaliadas com médias pouco mais elevadas que as demais, diferiram $(\mathrm{p} \leq$ $5 \%$ apenas da amostra 18 (comercial).

As amostras 6 e $7\left(4,78 \%\right.$ de $\left.\mathrm{K}_{\mathrm{c}} \mathrm{CO}_{s}\right)$ foram avaliadas como as de sabor adstringente mais forte (médias ao redor de 4), em relação às demais que apresentaram sabor adstringente mais "fraco" .

Os provadores não perceberam diferenças significativas ( $\mathrm{p} \leq 5 \%$ ) do gosto amargo, que foi avaliado próximo do "fraco" em todas as amostras analisadas. O gosto amargo é derivado das purinas e polifenóis do cacau, sendo um dos sabores básicos do chocolate, e podendo ser considerado indesejável quando em excesso, segundo LOPEZ \& McDONALD [5].

As avaliações do gosto salgado foram bastante próximas do "fraco", apenas a amostra 7 apresentou diferença significativa ( $\mathrm{p} \leq 5 \%$ ) com as demais (Tabela 4). A amostra 4 foi considerada a de gosto doce mais fraco, diferenciando-se significativamente ao nivel de $5 \%$ das demais, que o apresentaram próximo ao meio da escala.

Com relação ao corpo da bebida, apesar das amostras 6 e 7 terem recebido notas mais altas em relação às demais amostras, todas obtiveram médias ao redor de 5 .

Pelos resultados da ACP das bebidas achocolatadas (Figura 3) observa-se que os componentes principais $1 \mathrm{e}$ 2 dos atributos da bebida achocolatada explicam 45,84\% da variação total ocorrida entre as amostras.

Os atributos queimado, adstringente, alcalino e amargo contribuem com maior peso sobre a variabilidade associada ao eixo 1, e os atributos chocolate e solubilidade (positivamente) tiveram maior influência sobre o eixo 2, estando o atributo chocolate com maior peso.

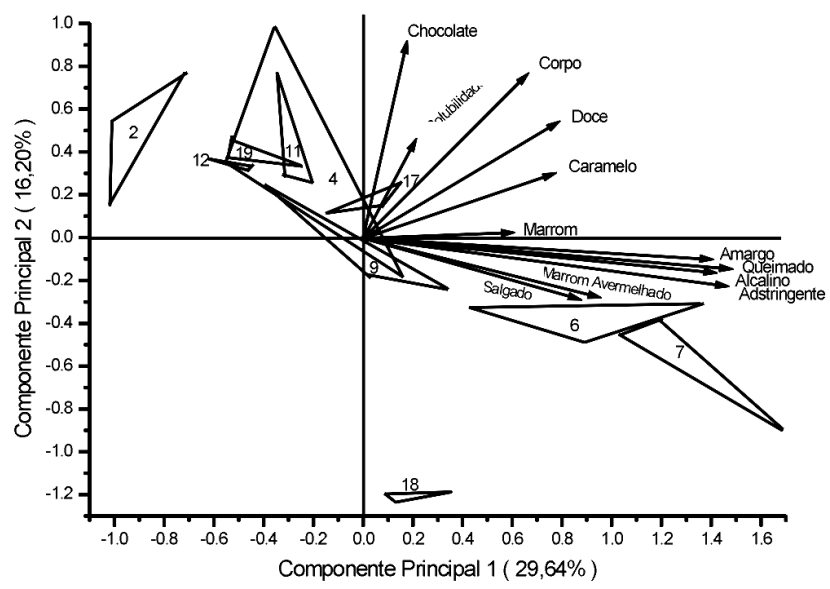

FIGURA 3 - Projeção bidimensional da análise de componentes principais das bebidas achocolatadas

As amostras 11, 12 e 19 (comercial) caracterizaramse pelo atributo sabor de "chocolate", a 17 com "chocolate" e "solubilidade" mostrando uma relação com o teor intermediário de $3,00 \% \mathrm{~K}, \mathrm{CO}$ empregado no processo de alcalinização destas amostras.

As amostras 6 e $7\left(4,78 \% \mathrm{~K} \mathrm{CO}_{s}\right)$ foram caracterizadas principalmente pelos atributos de gosto salgado, sabor alcalino, adstringente e queimado, e cor marrom avermelhada.

A amostra 18 (comercial) se destacou das demais por apresentar baixíssima intensidade de sabor de chocolate.

\section{4 - CONCLUSÕES}

Os três atributos gerados para a avaliação do aroma do pó de cacau e onze dos doze gerados para a avaliação das bebidas achocolatadas (excetuando o gosto "amargo") discriminaram as diversas amostras ao nivel de 5\% de probabilidade, sendo importantes para a análise da qualidade dos pós de cacau alcalinizados.

As avaliações dos aromas dos pós mostraram relação direta entre o alcalino e os teores de álcali, tempo e temperatura do processo e relação inversa com o de chocolate. Com relação ao aroma de queimado as amostras tratadas com maiores concentrações de $\mathrm{K}_{2} \mathrm{CO}_{s}$, tempo e temperatura o apresentaram com maior intensidade.

Nas bebidas achocolatadas observou-se relação inversa entre os teores de álcalis e a luminosidade da cor e relação direta com sabor "alcalino" e "queimado".

Todos os processos levaram à obtenção de amostras com solubilidade avaliada próximo ao "muito" e sabor "caramelo" e gosto "salgado" próximos ao "fraco".

O sabor de chocolate foi avaliado como mais "forte" nas amostras tratadas com menores concentrações de $\mathrm{K} \mathrm{CO}$.

Pelos resultados da análise de componentes princi- 
pais somente uma das amostras, de marca comercial, apresentou baixa intensidade de aroma e sabor de chocolate.

\section{5 - REFERÊNCIAS BIBLIOGRÁFICAS}

[1] ANDRES, C. New cocoa powder offers improved flavour. Food Processing, v.44, n.3, p.36-37, 1983.

[2] BISPO, E.S. Processo de alcalinização de "nibs" de cacau (Theobroma cacao L.) e avaliação da qualidade do pó por metodologia de superfície de resposta. Campinas, 2000. 120p. Tese (Doutorado em Tecnologia de Alimentos) Faculdade de Engenharia de Alimentos, UNICAMP.

[3] DAMÄSIO, M.H. \& COSTEL, E. Análises sensorial descriptivo generación de descriptores y selección de catadores. Rev. Agrquim. Tecnol. Alim., v.31, n.2, p.165-178, 1991 .

[4] KATTENBERG, R.H. The application of cocoa powder in chocolate confectionery. The Manufacturing Confectionery, v. 3, p. 73-83, 1995.

[5] LOPEZ, A. S.; McDONALD C. R. A definition of descriptors to be used for the qualification of chocolate flavours in organoleptic testing. Revista Theobroma, v.11, n.3, p. 209-217, 1981.
[6] MARTIN, J.R.; CHICHESTER, C.O.; MRACK, E.M.; SCAWEIGERT, B.S. Chocolate: Advances in Food Research. San Diego: Academic Press, 1987. 342 p.

[7] MEILGAARD, M.; CIVILLE, G. V.; CARR, B.T. Sensory Evaluation Techniques. 2.ed. Boca Raton: CRC Press, 1991. 354p.

[8] MINIFIE, B.W. Chocolate, cocoa and confectionery: science and technology. 3.ed. New York: AnAv, 1989. 904 p.

[9] MOSKOWITZ, H.R. Product testing and sensory evaluation of foods: marketing and $R \& D$ approaches. Westport, Food \& Nutrition Press, 1983. 605 p.

[10] SAS Institute. SAS User's Guide: statistics. Cary, USA: SAS Inst., 1993.

[11] SCHENKEL, H.J. Colour formation in the Dutch processing of cocoa. Manufacturing Confectioner, v.53, n. 8, p.26-28, 1973.

[12] STONE, H. SIDEL, J. L. Sensory Evaluation Practices. 2ed. San Diego: Academic Press, 1993.338p.

[13] TERINK, J.L.; BRANDON, M.L. Alkalized cocoa powders and foodstuffs containing such powders. Cacaofabrik de Zaam BU; Int $\mathrm{Cl}^{3}$ A23G 1/102 NL. 8102377. 14 maio 